\title{
A Novel Fixed-Time-Convergent Sliding Mode Technology Using Improved Quantum Particle Swarm Optimization for Renewable Energy Inverters
}

\section{En-Chih Chang}

Department of Electrical Engineering, I-Shou University, No. 1, Sec. 1, Syuecheng Rd., Dashu District, Kaohsiung City 84001, Taiwan; enchihchang@isu.edu.tw; Tel.: +886-7-6577711 (ext. 6642); Fax: +886-7-6577205

Received: 31 December 2019; Accepted: 2 February 2020; Published: 4 February 2020

\begin{abstract}
This paper describes a robust feedback technique involving novel fixed-time-convergent sliding mode technology (NFTCSMT) using improved quantum particle swarm optimization (QPSO) to obtain high-performance renewable energy inverters. Customary SMT encounters long time convergence towards the origin and the influence of the dithering. The NFTCSMT can rapidly impel system-following movement to approach the sliding manifold and effectively accelerate the convergence speed to equilibrium states. However, the NFTCSMT cannot easily select the global optimum of the controller parameters subject to large parameter changes and nonlinear interventions, leading to the dither phenomenon/steady-state error still being caused. The dither inflicts decreased control accuracy, high voltage harmonics, major harm in relation to switching components, and great thermal losses in power electronic converters. The improved QPSO including the unique property of a random compression/expansion factor is used to find optimal parameters of the NFTCSMT in practical applications, for the reason that it importantly mitigates the dither and amends convergent speed as well as guaranteeing global convergence. The presented alliance amid NFTCSMT and improved QPSO achieves faster response time and singularityless, and also yields high-accuracy tracking and dither attenuation. The robust stability using Lyapunov theorem of the suggested system has provided precise mathematical derivations. Simulations show that the suggested controller offers less than $0.1 \%$ voltage THD (total harmonic distortion) which exceeds IEEE standard 519 under heavily distorted rectifier loads, and less than $10 \%$ voltage dip which surpasses IEEE standard 1159 during step load transients. Experimental tests of an algorithmically controlled laboratory prototype $(1 \mathrm{~kW}, 110 \mathrm{Vrms} / 60 \mathrm{~Hz})$ of a renewable energy inverter (REI) based on digital signal processing manifest less than $0.05 \%$ voltage THD in the face of great inductor-capacitor alterations, and less than $10 \%$ voltage dip in the face of transient load scenarios.
\end{abstract}

Keywords: novel fixed-time-convergent sliding mode technology (NFTCSMT); improved quantum particle swarm optimization (QPSO); global convergence; faster response time; renewable energy inverter (REI)

\section{Introduction}

Renewable energy inverters have been largely used in photovoltaic (PV) array power production, wind-driven power generation, and hydrogen-based fuel-cell power production [1-3]. A PV power production system is capable of turning sunshine into serviceable electricity. The usual PV power generation system comprises a solar array, DC (direct current)-to-DC (direct current) converter, DC (direct current)-to-AC (alternating current) inverter, and connected loading. It is designed to generate maximum power for the sake of transmitting it to the connected loading. Such a configuration exists in two energy converters that are seen as a dual-stage system. Both maximum power point tracking 
(MPPT) and output-response regulation are realized by the DC-to-DC converter. The DC-to-AC inverter converts $\mathrm{DC}$ power yielded by a solar array into an $\mathrm{AC}$ power supply delivered to a home or to the grid. In addition, one type of single-stage DC/AC inverter can merge both DC-to-DC and DC-to-AC stages. Among various topologies of power converters in renewable energy applications, the low pass LC inverter is the key component for operation interface between the renewable energy source and electrical AC grid. Even under non-linear loads, REIs should maintain lower amounts of harmonic voltage distortion in the high quality AC output response, which can only be achieved by using feedback control techniques [4-6].

Sliding mode Technology (SMT) is robust in the face of parametric changes and external interruptions. The control methodology of the DC-to-AC inverter has shown that the SMT offers agreeable design alternatives [7-10]. Based on the dissimilar control object as well as entire and partial system-state data, an exhaustive control design for DC-to-AC inverters can be developed. However, the algorithm employs customary SMC and has arduous realization, thus displaying the distortion of the output voltage during steady-state and a transient state [11]. The sliding-mode tracking controller with the constant switching-frequency method has been recommended to control the output of the DC-to-AC inverter. In this situation, the consequent output-voltage makes a trade-off between linear and nonlinear loading [12]. The variable structure scheme combined with a Lyapunov candidate methodology is designed for gaining a robust response of an island microgrid. Such a control method necessitates an involved circuit design and yields the unwanted dithering phenomenon [13]. An integral sliding mode controlled DC-to-AC system is presented. Though the steady state response has been resolved as a consequence of an integral compensation, the system behavior does not exactly provide the desired switching manifold. The output waveform of a nonlinear condition is highly distorted [14]. Additionally, a second-order SMC has been adopted for grid-tied energy systems, but the rapid dynamic performance cannot be seen, and a large amount of dithering still exists [15].

As noted above, these customary SMT schemes may face the problems of long convergence time and harsh dithering. In terms of the trajectory tracking control, the invariance characteristic of the customary SMT only can be ensured with a sliding duration, and the state behavior of the arriving phase is frequently disturbed by the external uncertainties/the change of the internal parameters. Earlier investigations involving time-varying SMT, intelligent sliding mode-based observer, or enhanced SMC with an exponential reaching law, have attempted to accelerate arrival time and moderate tracking error. The time-varying SMT can shorten the arriving phase, where the sliding manifold is shifted and/or rotated. Though the beginning condition can be arbitrarily specified in the normal system, it does not satisfy the uncertain situation [16]. The disturbance observer has less dithering, however a longer arriving time in the arriving stage also occurs [17]. The exponential reaching law-based SMT was developed for the application of renewable energy. This enhanced methodology can accelerate attainment of the reaching time, but there is no decreasing trend of the dither around the sliding manifold [18].

It was plain that in these latter years, terminal sliding-mode variable structure control (TSMVSC), which generates limited convergent response and singularityless, has provided another classification of the SMT. This TSMVSC is continually used to obtain the achieved contributions [19-23]. In spite of the TSMVSC's behavioral convergency within a limited speed while satisfactorily maintaining the SMC's customary robustness, it reveals the perplexity of the dithering. In practice, it is hard to be aware of the system parametric changes, external load disturbances, and unmodeled dynamics. If the event of there being a large/small system uncertainty limit, the dither or steady-state error yields, which is unable to guarantee the existence of an invariant sliding mode. Much research uses self-tuning control methods to solve the dither influence issued from bound ambiguity [24-29]. These can abate a few dithering as well as steady state error, thereby impeding the enhancement of the dynamics and stationary state [30-36]. The TSMVSC parametric decision in particular is faced with the difficult choice in order to obtain the best gain values under significant parameter changes and vague interruptions [37]; meanwhile, using a resulting state convergence speed to reach zero is not fast enough or accurate. 
For the sake of surmounting the vexations of the dither as well as steady state error in the TSMVSC, and more importantly accelerating the system's convergence speed to reach the equilibrium point, this paper proposes a novel fixed-time-convergent sliding mode technology (NFTCSMT) conjoined using improved quantum particle swarm optimization (QPSO). The NFTCSMT is effective with respect to having a faster convergence speed, short dynamic response time, and higher tracking accuracy. Moreover, in the coming era of artificial intelligence, the QPSO is widely used to solve complex optimization problems because of its strong global search ability, faster execution time, and high quality solutions [38-41]. Therefore, the improved QPSO encompassing unique feature (randomized compression/expansion factor) is used to find the best value of NFTCSMT parameters, which can remarkably enhance the control accuracy with the dithering removal and evade inefficient, tedious, and time-consuming adjustments. In contrast to the customary TSMVSC/SMC without an optimal algorithm, this suggested controller offers another choice and possible suggestions. Despite the eventual response in the suggested system without exceeding the outcome of the harmonic-distortion-suppression from the late studies, the simplicity, speed, and efficiency of the algorithm are obviously superior to the existing research results. The suggested controller does enhance the TSMVSC method and provides the optimized adjustment for determining the controller parameters. Combining NFTCSMT with improved QPSO, a closed-loop controlled inverter system is designed to produce a low THD and rapid dynamic response under various loading cases. The verification of numerical simulations and experiments has illustrated the ascendancy of the suggested REI.

\section{Circuit Modeling of REI}

Figure 1 exemplifies the classically used framework in a REI. The major operating components of an inverter involve four semiconductor switching devices assembly from n-channel metal-oxide-semiconductor field-effect transistors, an inductor-capacitor $\left(L_{r} C_{r}\right)$ low-pass filter, and a resistance loading $R_{l}$. Its DC-link voltage notates $V_{d c}$ and the output-voltage represents $v_{a c}$.

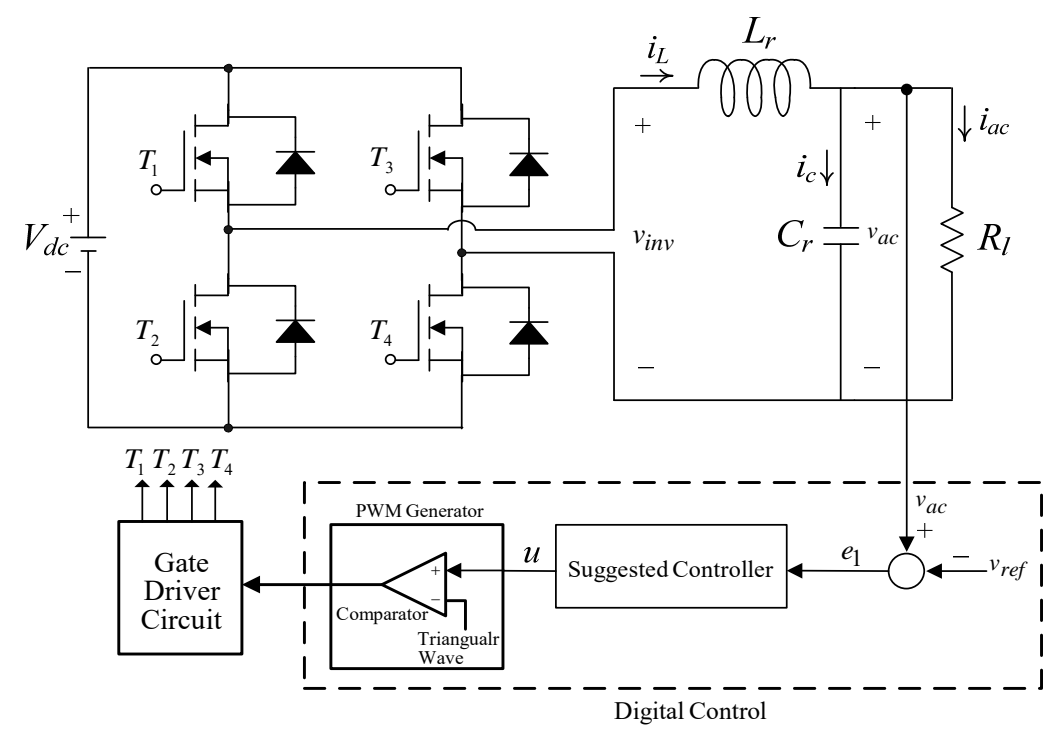

Figure 1. Framework of renewable energy inverter.

Let $\dot{v}_{a c}$ be the derivative of $v_{a c}$ and select the system state vector as $\left[\begin{array}{lll}x_{1}=v_{a c} & x_{2}=\dot{v}_{a c}\end{array}\right]^{T}$, then the state equation becomes:

$$
\left[\begin{array}{l}
\dot{x}_{1} \\
\dot{x}_{2}
\end{array}\right]=\left[\begin{array}{cc}
0 & 1 \\
-a_{1} & -a_{2}
\end{array}\right]\left[\begin{array}{l}
x_{1} \\
x_{2}
\end{array}\right]+\left[\begin{array}{l}
0 \\
b
\end{array}\right] u
$$


where $a_{1}=1 / L_{r} C_{r}, a_{2}=1 / R_{l} C_{r}, b=\bar{k}_{p} / L_{r} C_{r}, \bar{k}_{p}$ stands for inverter proportional, and $u=v_{\text {inv }} \approx$ $\left(\Delta T_{s} / T_{s}\right) \cdot V_{d c}$. The controller output $u$ infers $\kappa V_{d c}$ in which the switching duty ratio $\kappa$ ranges between -1 and 1 . Note that the $u$ is determined via the controller and its actual conversion by a pulse width modulation (PWM) method [42,43] yields the switching duty ratio. The control signal is also equal to the average output-voltage of the inverter bridge $v_{i n v}$ containing a width $\Delta T_{S}$ centered at sampling interval $T_{s}$, and thus does not involve a corresponding PWM conversion process as a result of the control law.

The purpose of a closed-loop system involves deriving the $u$, enabling output voltage $v_{a c}$ to track the expected sine waveform $v_{r e f}=V_{m} \sin (\omega t)$ with a maximum value as well as an angular frequency $\omega$ in radians per second. Then, the corresponding error state-variables $\left(e_{1}\right.$ and $\left.e_{2}\right)$ can be formulated as

$$
\begin{aligned}
& e_{1}(t)=v_{a c}(t)-v_{r e f}(t) \\
& e_{2}(t)=\dot{v}_{a c}(t)-\dot{v}_{r e f}(t)
\end{aligned}
$$

where $e_{1}$ denotes following error and $e_{2}$ signifies the derivative of $e_{1}$.

The error state equation for the REI is then derived as

$$
\left[\begin{array}{l}
\dot{e}_{1} \\
\dot{e}_{2}
\end{array}\right]=\left[\begin{array}{cc}
0 & 1 \\
-a_{1} & -a_{2}
\end{array}\right]\left[\begin{array}{l}
e_{1} \\
e_{2}
\end{array}\right]+\left[\begin{array}{l}
0 \\
b
\end{array}\right] u+\left[\begin{array}{c}
0 \\
-a_{1} v_{r e f}-a_{2} \dot{v}_{r e f}-\ddot{v}_{r e f}
\end{array}\right]
$$

where $p=-a_{1} v_{r e f}-a_{2} \dot{v}_{r e f}-\ddot{v}_{r e f}$ is a perturbation which has a limit of $\|p(\cdot)\|<\delta$, here $\delta$ is a positive constant. The controller output $u$ displayed in (4) implies that the designer calls for ensuring dynamic errors $e_{1}$ and $e_{2}$ driven to the origin. In fact, the suggested NFTCSMT is looked upon as an efficient method with shorter-time convergent characteristics. The NFTCSMT-based REI has a singularity-free faster-converging speed, strong dynamic as well as stationary states, and fixed reachable-time stabilization. In conditions with unexpected step-loading variations/serious nonlinear meddling, the NFTCSMT inverter is prone to dither or following error in a stationary state, conducing to erroneous responses. Many explorations attempt to obtain smaller amounts of dithering or to minimize following errors. But then, if viewed from another angle, the practical application of artificial intelligence methods provides very interesting subjects among the many areas of applied sciences and industrial technology. Based on this motivation, it would be a good idea to introduce a globally-optimal methodology into NFTCSMT design to obtain the best solution. Such a practice offers the system with the support of more robust adaptability, while even attracting the readership allied to renewable energies as an optional literature resource. As such, the NFTCSMT combining improved QPSO algorithm is presented to perform dither/steady-state error attenuation of customary TSMVSC/SMC, providing more accurate tracking. The REI employing this suggested control design is able to produce lower total harmonic distortion (THD) percentages with regard to vague interventions.

\section{Suggested Controller Design}

\subsection{Problem Statement}

First, the unsatisfactory response of a classical handling system by the TSMVSC is exposed, and then the suggested controller is designed.

Suppose a second order non-linear dynamic as follows

$$
\left\{\begin{array}{l}
\dot{z}_{1}=z_{2} \\
\dot{z}_{2}=f_{z}\left(z_{1}, z_{2}\right)+b_{z} u+p_{z}\left(z_{1}, z_{2}, t\right)
\end{array}\right.
$$

where $z_{1}$ and $z_{2}$ are state variables, $f_{z}(\cdot)$ represents known nonlinearity, $b_{z}$ means control gain, $u$ stands for control law, and $p_{z}(\cdot)$ connotes an unknown extraneous perturbation. 
Customary TSMVSC can be expressed as

$$
\sigma=z_{2}+\gamma\left|z_{1}\right|^{\rho} \operatorname{sign}\left(z_{1}\right)
$$

where $\gamma$ is a positive real number, $\rho$ bounds between 0 and 1 , and $\operatorname{sign}(\cdot)$ symbolizes the signum function. Despite the system's limited time stability existed in TSMVSC, its efficiency and convergence rate decrease, in which case the initial dynamical behavior removes the system from an equilibratory position. A fast TSMVSC is developed to solve this problem below.

$$
\sigma=z_{2}+\lambda z_{1}+\gamma\left|z_{1}\right|^{\rho} \operatorname{sign}\left(z_{1}\right)
$$

where $\lambda$ points to a positive real number. As $z_{1}$ is removed from the origin, the state property passes into exponential stability. As $z_{1}$ approaches the origin, this fast TSMVSC can help accelerate the system's convergent speed, thus resulting in limited time reachability. However, if $z_{2} \neq 0$ while $z_{1}=0$, the control input will appear as a singularity problem in practice.

A nonsingular TSMVSC has been designed to overcome the singularity problem as follows:

$$
\sigma=z_{1}+\eta^{-1} z_{2}^{h / g}
$$

where $\eta>0$, and $g$ and $h$ imply positive odd numbers which have a limits at the inequality $g<h<2 g$. The nonsingular TSMVSC displays a quicker convergent speed when the system state is removed from the equilibratory position, but it causes a slower convergent time when the system state approaches the equilibratory position.

\subsection{Derivation and Analysis of NFTCSMT Combined with Improved QPSO}

For the reforms of the above-depicted TSMVSC shortcomings, the NFTCSMT with the error dynamics (4) is developed below and configured to assure rapider convergence and more accurate tracking control without singularities.

$$
\sigma=e_{1}+\mu^{-1}\left|e_{1}\right|^{n / m} \operatorname{sign}\left(e_{1}\right)+\xi^{-1}\left|e_{2}\right|^{w / v} \operatorname{sign}\left(e_{2}\right)
$$

where $\mu>0, \xi>0$, and $m, n v, w$ are positive odd numbers, $1<w / v<2$, and $w / v>n / m$. Also, a sliding-mode reaching equation $\dot{\sigma}=\left[-\tau_{1} \sigma^{k_{1} / j_{1}}-\tau_{2}|\sigma|^{k_{2} / j_{2}} \operatorname{sign}(\sigma)\right]\left|e_{2}\right|^{w / v-1}$, here $\tau_{1}>0$ and $\tau_{2}>0$, and $j_{1}, j_{2} k_{1}, k_{2}$ are positive odd numbers, which can be used to derive the control law.

The NFTCSMT's feedback control law can be designed as

$$
\begin{array}{r}
u(t)=-\frac{\xi g}{h b}\left\{\left[\tau_{1} \sigma^{k_{1} / j_{1}}+\tau_{2}|\sigma|^{k_{2} / j_{2}} \operatorname{sign}(\sigma)\right]+\left|e_{2}\right|^{2-w / v} \operatorname{sign}\left(e_{2}\right)\right. \\
\left.+\frac{n}{\mu m}\left|e_{1}\right|^{n / m-1}\left|e_{2}\right|^{2-w / v} \operatorname{sign}\left(e_{2}\right)\right\}+\frac{1}{b}\left(a_{1} e_{1}+a_{2} e_{2}\right)
\end{array}
$$

Proof. The candidate function of the Lyapunov is chosen as

$$
V=\sigma^{2} / 2
$$

Along the dynamic behavior (4) with the law of the control (11), the derivative of the $\dot{V}$ produces

$$
\begin{aligned}
\dot{V} & =\sigma \dot{\sigma} \\
& =\sigma\left(\dot{e}_{1}+\frac{n}{\mu m}\left|e_{1}\right|^{n / m-1} \dot{e}_{1}+\frac{h}{\xi g}\left|e_{2}\right|^{w / v-1} \dot{e}_{2}\right) \\
& =-\left(\tau_{1} \sigma^{k_{1} / j_{1}+1}+\tau_{2}|\sigma|^{k_{2} / j_{2}+1}\right)\left|e_{2}\right|^{w / v-1}
\end{aligned}
$$


This result is due to the presence of $\sigma \neq 0$ and $e_{2} \neq 0$, when the $\dot{V}$ is less than zero. It is deduced that the Lyapunov stability derived from (12) converges to the equilibratory position for a more rapid fixed/limited time. The system state (4) then contributes a more rapid fixed/limited time convergency to the equilibratory position. However, form realistic design of the REI, the dither/following error repeatedly emerges from NFTCSMT. The reason this occurs is that the load condition is variable, and if the load is severely uncertain, the system (4) will not be able to achieve an exact tracking performance. The output response of the REI does not identically pursue the reference signal waveshape. The control law (10) is modified by adding an improved QPSO methodology, which inhibits dither/following error from appearing throughout REIs. The searching out of the NFTCSMT best parameter values in (10) is very essential to preserving good inverter performance in transiency and stationary states. Thereby to eradicate dither/following error, ward off tedious time-consuming and inefficient computations, and gain the global best solutions, the NFTCSMT best parameter values are determined by the improved QPSO methodology. The REI with the associating use of the improved QPSO and NFTCSMT can achieve a faster fixed-time convergent stability, so the reachability of a closed-loop zero-following error is achieved within a very short duration. Comparing with the improved QPSO algorithm, the traditional PSO algorithm cannot converge to the global best point with probability of 1 , that is, it does not have global convergence. The speed of a single particle has an upper limit, and its search space is limited, which cannot completely embrace a possible solution region with a globally limited searching ability. Oppositely, the improved QPSO algorithm as a global optimization metaheuristic approach provides a completely random iterative equation, and no longer needs the velocity vector, thus achieving quick convergence speed and enhancing search efficiency and global optimization. There is no fixed trajectory when the particles move in the quantum space, and the parameters of the entire iterative equations are fewer and easier to control, therefore enabling searching for the best particles solution among the entire possible region. The representative expressions in (13) and (14) of the improved QPSO algorithm are formulated as follows, and notably (14) with randomized compression/expansion factor improves the convergent speed and ensures a global convergency, as compared with the customary PSO.

$$
\begin{aligned}
& \psi_{i}^{k}=\phi^{k} \psi_{\text {pbest }, i}^{k}+\left(1-\phi^{k}\right) \psi_{g b e s t, i}^{k} \\
& X_{i}^{k+1}=\psi_{i}^{k} \pm \beta^{k}\left|Q^{k}-X_{i}^{k}\right| \ln \left(\frac{1}{\Theta^{k}}\right)
\end{aligned}
$$

where $\beta^{k}=\beta_{b}+\varepsilon$ is the compression/expansion factor, $\beta_{b}$ stands for the basic compression/expansion factor, and $\varepsilon$ represents a random number between 0 and $1, \phi^{k}$ and $\Theta^{k}$ signify random numbers including uniform distribution between 0 and 1 , and $Q^{k}$ indicates the mean best location in the population environment of entire particles. The steps of improved QPSO are described in detail as follow: Step 1: Execute the Initiation of the particles associated with random locations. Step 2: Settle the mean best location $Q^{k}$ in entire particles. Step 3: For the purpose of each particle usage, appraise the required objective function, and then make a comparison with regard to the prior best value of the particle. While the present value is smaller than the prior best value, determine the current value to be the best value. Step 4: Determine the minimization of the present global location in the best location of the particles. Step 5: Draw a comparison amid the present global location and the prior global location. When the present global location is smaller than the prior global location, treat the present global location as the global location. 6. Regarding the range of each particle, gain a random point amid $\psi_{i}^{k}$ and $\psi_{\text {gbest }, i}^{k}$ by using Equation (13). Step 7: Acquire the new location through Equation (14). Step 8: Reiterate Steps 2 to 7, pending the satisfaction of the termination condition.

The front derived consequences are sketched to dissect and clarify the diversity of the response amid the customary TSMVSC, as well as the suggested controller. The customary TSMVSC actually includes the ingredients of the equivalent control, manifesting the singularities and sliding control while disclosing the dither/following error. The suggested controller has been developed to vanquish 
the singularities, yet a switching gain of the customary TSMVSC is usually higher than/equal to vague interruptions. Thereby, the difficulty of the sliding control ingredient in the customary TSMVSC is defeated by both the switching gain existing in the suggested controller and the improved QPSO. In the case of an unexpectedly greater limit of parameter variations/serious nonlinear meddling, an exorbitantly cautious switching-gain yields a great amount of dither in the customary TSMVSC. The improved QPSO can atone for sliding-manifold magnitude overstepping the limit layer breadth on purpose to abate both switching-gain magnitude and dither occurring in sliding control ingredient. In a nutshell, an exorbitantly cautious switching-gain for the suggested controller becomes two ingredients associated with sliding control and improved QPSO. In case a greater limit of parameter variations/serious nonlinear meddling is unexpected, the smaller gain amplitude occurs in the suggested controller, as compared with the customary TSMVSC, thus relieving the level of the dither, subject to vague interruptions.

\section{Results and Discussion}

For specified good performance (less than 5\% voltage THD recommended in IEEE standard 519 , and smaller than $20 \%$ voltage dip prescribed by IEEE standards 1159) and strong robustness (finite-time global Lyapunov stability), the numerical simulations as well as experiments are afforded for the confrontation amid the customary TSMVSC and the suggested controller. The parameters of the presented circuit are given in the following $V_{d c}=200 \mathrm{~V}, v_{a c}=110 \mathrm{~V}_{\mathrm{rms}}, f_{a c}=60 \mathrm{~Hz}$, $v_{\text {ref }}(t)=110 \sqrt{2} \cdot \sin (2 \pi \cdot 60 \cdot t), f_{s w}=18 \mathrm{kHz}, L_{r}=0.125 \mathrm{mH}, C_{r}=20 \mu \mathrm{F}$, rated load $=12 \Omega$. When the maximum triggering angle is limited to ninety degrees for step-load variations from no loading to full loading, two same REIs managed through the customary TSMVSC as well as the suggested controller are tested to demonstrate the transient performance. The simulated output waveforms of REIs have been depicted as Figures 2 and 3, individually. With the cautious scrutiny at Figure 3, the suggested controller can compensate the instantaneous voltage dip very quickly and establish a limited system state convergence time, so that the control is more precise. Figure 4 displays the simulated output waveform of a REI controlled by a traditional TSMVSC under a non-linear load condition (diode bridge rectifier feeding a capacitor $100 \mu \mathrm{F}$ connected in parallel with a resistance loading $30 \Omega$ ). Obviously, the output voltage is a contorted sine waveform with a high \%THD of $26.82 \%$. Figure 5 shows the simulated output waveform of the suggested controller under same non-linear load condition (full-wave bridge rectifier connecting a filter capacitor $100 \mu \mathrm{F}$ across a resistive loading $30 \Omega$ ). Despite having high sharp-wave current yields, the output voltage waveform is very near to the sinusoidal reference voltage waveform (low \%THD of $0.07 \%$ ). It can be seen that the suggested REI has a stronger performance than the REI controlled by the traditional TSMVSC. Table 1 gives simulation confrontation for dips and THD percentage values in output-response under step-loading variations from no loading to full loading and nonlinear loading. 


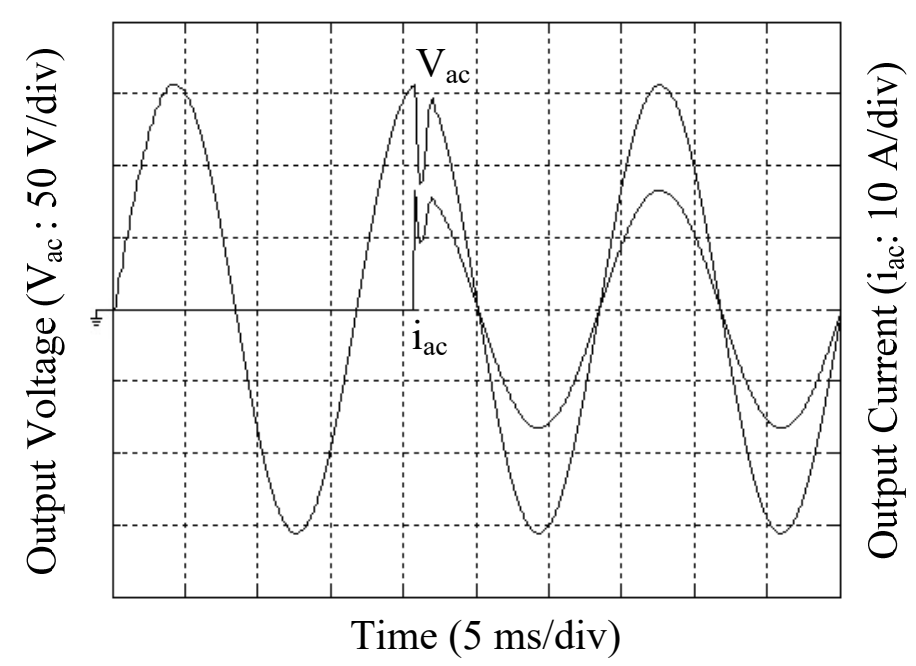

Figure 2. Simulation response using the customary TSMVSC subject to step-loading variation (form no loading to full loading).

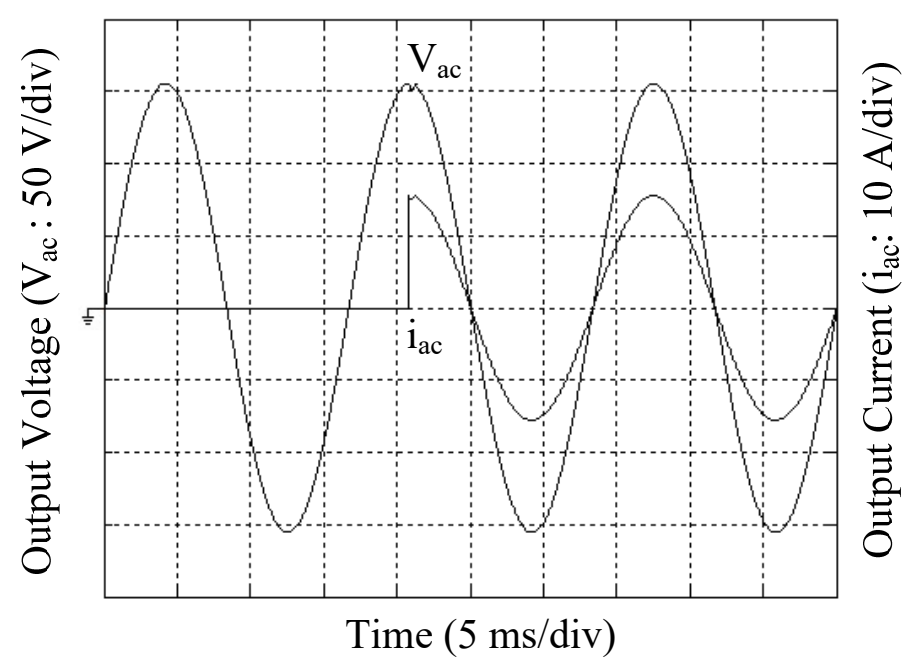

Figure 3. Simulation response using the suggested controller subject to step-loading variation (form no loading to full loading).
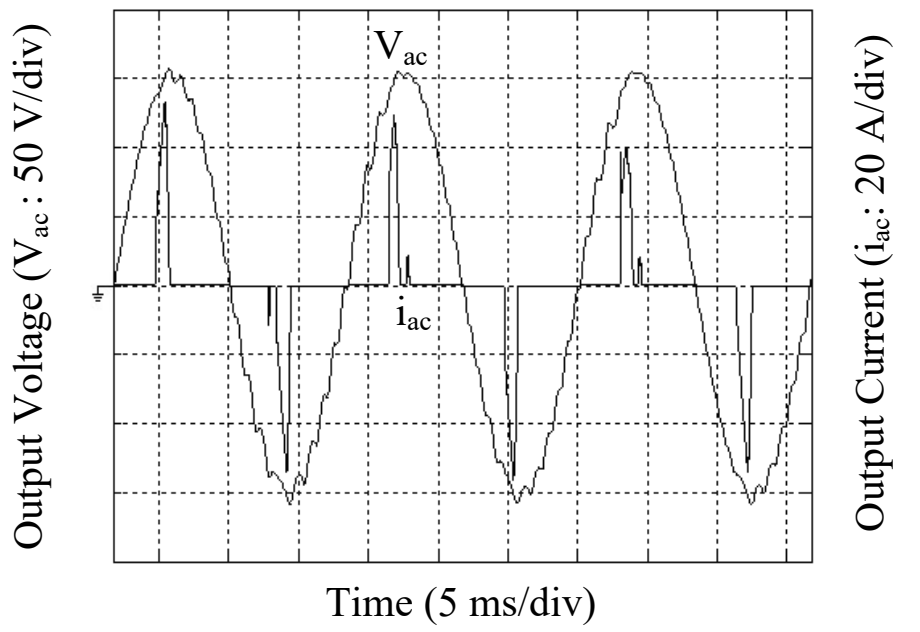

Figure 4. Simulation response using the customary TSMVSC subject to nonlinear loading. 


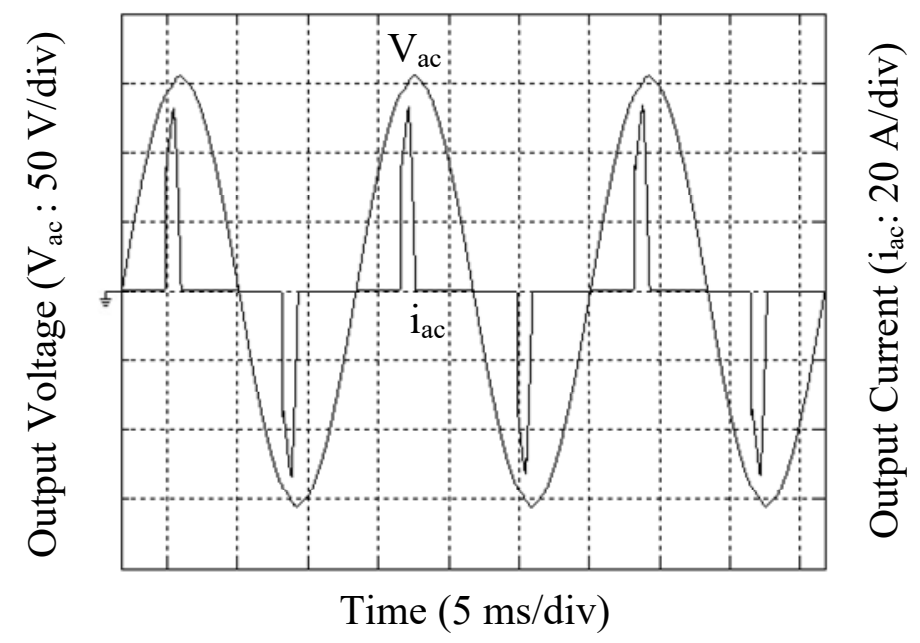

Figure 5. Simulation response using the suggested controller subject to nonlinear loading.

Table 1. Simulation response for dips and THD percentage values subject to step-loading variations as well as nonlinear loading.

\begin{tabular}{lcc}
\hline & Simulation Results & \\
\hline \multirow{2}{*}{ CustomaryTSMVSC } & Step-loading variations (Voltage dip) & $49 \mathrm{~V}_{\mathrm{rms}}$ \\
& Nonlinear loading (\%THD) & $26.82 \%$ \\
\hline \multirow{2}{*}{ Suggested controller } & Step-loading variations (Voltage dip) & $4 \mathrm{~V}_{\mathrm{rms}}$ \\
& Nonlinear loading (\%THD) & $0.07 \%$ \\
\hline
\end{tabular}

Also in terms of digital controller based on dSPACE DSP (digital signal processing) implementation, a $1 \mathrm{~kW}$ lab prototype of a REI has been configured as Figure 6 . The inference circuit functions as a medium between dSPACE DSP and REI. Amid the control circuit and the power-stage circuit employ four high-speed optocouplers (PC923) to guarantee electrical isolation. The model number of four power metal-oxide-semiconductor field-effect transistors is called IRF460, and the isolated amplifier (AD202) aims at voltage sensing. Figure 7 shows the experimentation response for a REI governed via the customary TSMVSC with triggering angle of ninety degrees under step-loading variations from no loading to full loading. It can be seen from the figure that the instantaneous voltage dip does not recover quickly, and the controller's compensation ability to the instantaneous voltage dip is obviously poor. Figure 8 illustrates the experimental output waveforms for a REI when this suggested control law is used at ninety degrees, triggering the angle subject to step-loading variations from no loading to full loading by circumspectly looking at the recoupment of the instantaneous voltage dip, the fast transient-response speed can be acquired in a very short time. The compensation ability of an exterior loading interruption is better than the customary TSMVSC. Figures 9 and 10 exhibit the experimental output voltages for two same REIs handled through the customary TSMVSC as well as the suggested controller when the filter parameters are changed ( $5 \%$ to $100 \%$ respecting nominal parameter quantity subject to resistance loading $12 \Omega$ ). By using the suggested controller, the output voltage displayed in Figure 10 achieves a strong tolerability for parametric variation. Nevertheless, a periodic output-voltage distortion represented in Figure 9 can be seen clearly. Table 2 compares the experimentation response in dips and THD percentage values subject to step-loading variations as well as inductor-capacitor alterations. 


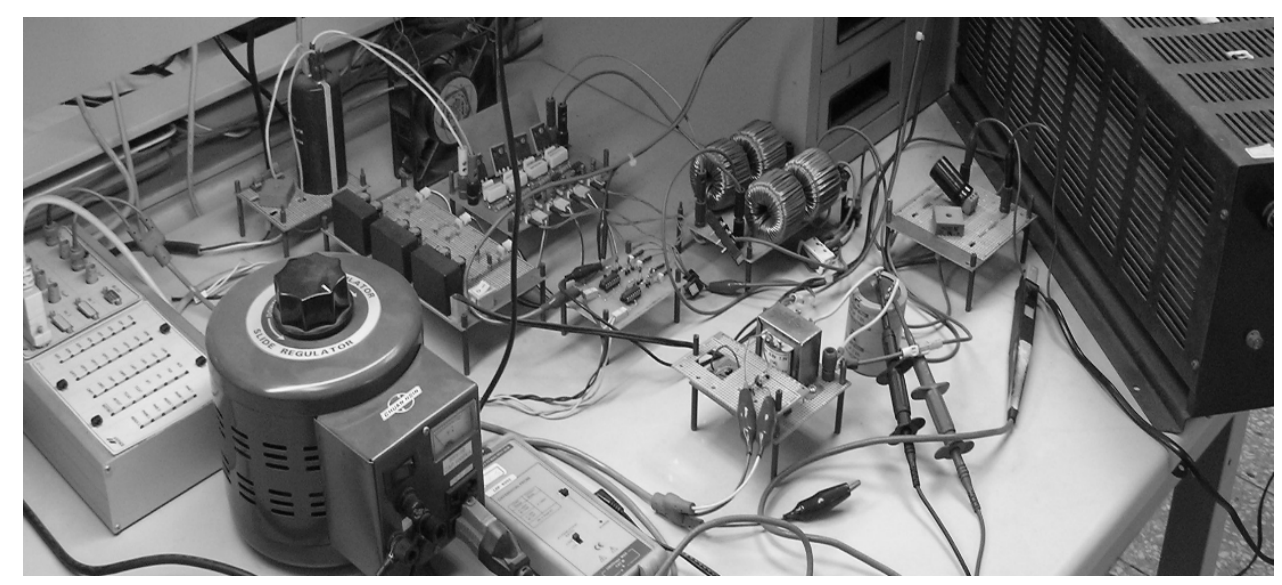

Figure 6. Suggested control algorithm for DSP-based realization.

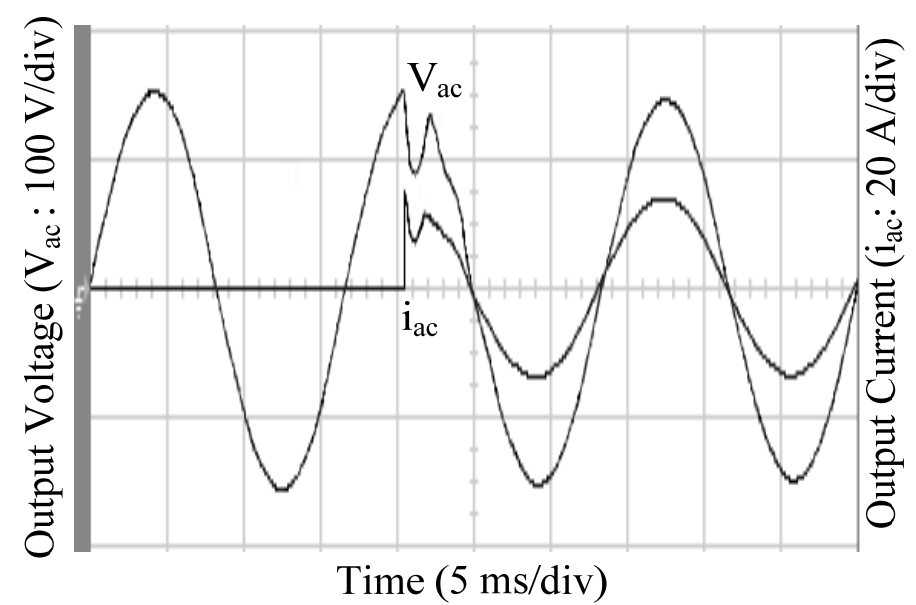

Figure 7. Experimentation response using the customary TSMVSC subject to step-loading variations (form no loading to full loading).

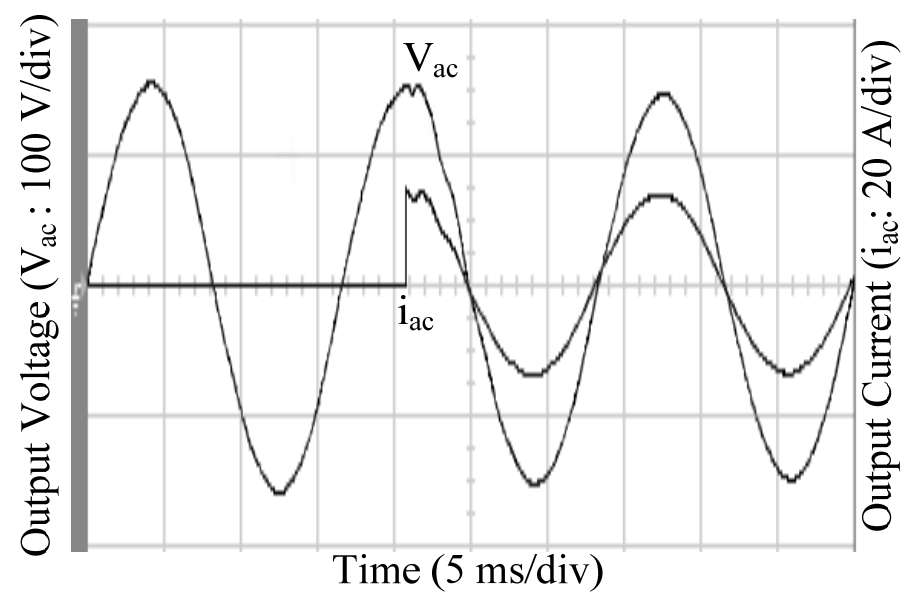

Figure 8. Experimentation response using the suggested controller subject to step-loading variations (form no loading to full loading). 


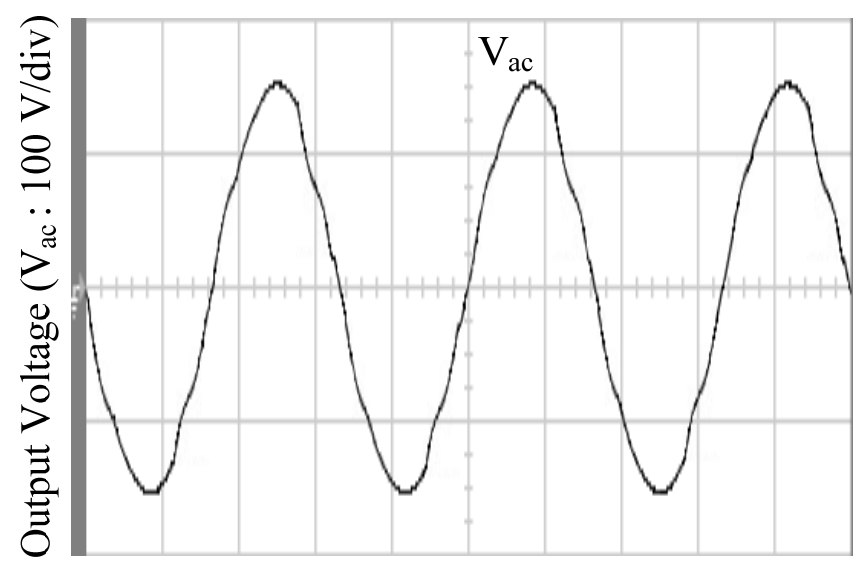

Time $(5 \mathrm{~ms} / \mathrm{div})$

Figure 9. Experimentation response using the customary TSMVSC subject to inductor-capacitor alterations.

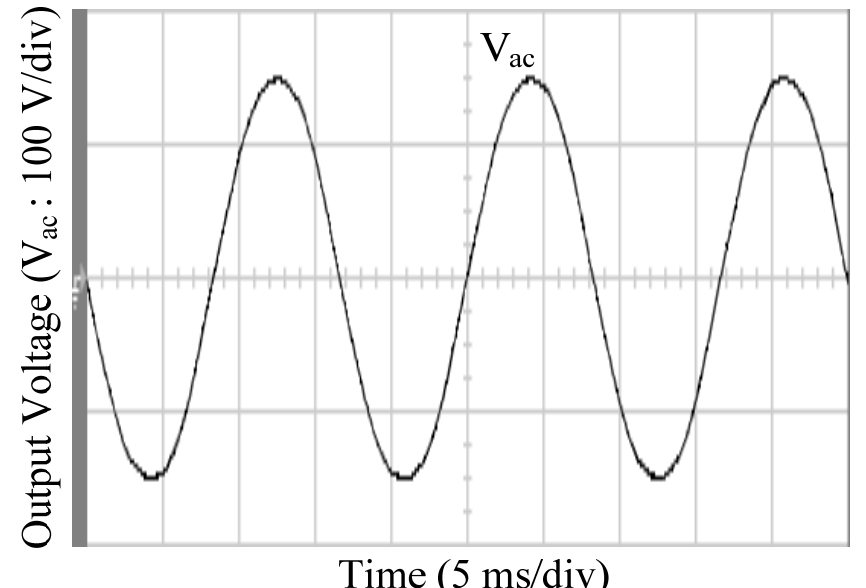

Figure 10. Experimentation response using the suggested controller subject to inductor-capacitor alterations.

Table 2. Experimentation response for dips and THD percentage values subject to step-loading variations as well as inductor-capacitor alterations.

\begin{tabular}{lcc}
\hline & Experimental Results & \\
\hline \multirow{2}{*}{ CustomaryTSMVSC } & Step-loading variations (Voltage dip) & $48 \mathrm{~V}_{\text {rms }}$ \\
& Inductor-capacitor alterations (\%THD) & $16.63 \%$ \\
\hline \multirow{2}{*}{ Suggested controller } & Step-loading variations (Voltage dip) & $7 \mathrm{~V}_{\mathrm{rms}}$ \\
& Inductor-capacitor alterations (\%THD) & $0.03 \%$ \\
\hline
\end{tabular}

A synopsis relative to numerical simulations and experimentation effects communicates the collation amid the customary TSMVSC and the suggested controller. On practical views of the realization, the switching-gain in the customary TSMVSC is generally in direct ratio to the greater limit of parameter variations/vague interventions. A sizable switching-gain aims at stabilizing the customary TSMVSC inverter in case of serious parameter vagueness as well as exterior interruption. Such treatment frequently incurs intolerable intense dithering. The improved QPSO is devoted to discovering switching gain adaptively, which acquires a decrement in dither as well as an augmentation in transiency and steady state response. Owing to the less calculational complicacy, the improved QPSO and the adopted DSP including a 3 ms conversion time is fast enough to meet the computational requirements of the algorithm, and the confrontation of optimization results are plotted in Figure 11 among the customary TSMVSC, NFTCSMT, and suggested controller. As a consequence, the suggested 
controller uncovers the mitigation of the dither, the minimization of the following-error and the exception of the intervention. Based on an intention of the prospective proceeded study, the publication reviews of extra robust schemes, such as the deadbeat control, repetitive control, $\mathrm{H}$-infinity method, and mu-synthesis approach are provided below. A deadbeat control by using on predictive modeling has been presented for the application of the neutral-point-clamped inverter. The developed methodology has low sensitivity to interior parameter changes and exterior loading interruption. A reduction of calculation complication can be achieved because there is no use of optimal iteration. The presented inverter unfolds zero following error and expeditious transiency [44]. The closed-loop design of the repetitive method is proposed for power inverters, offering the removal of following error and duration disturbance. It displays the maximization of system robust stability subject to loading changes and vagueness. The developed inverter yields low total harmonic distortion as well as speedy dynamics in the presence of severe load variations [45]. The H-infinity method with the help of the backstepping design strives to handle PV grid-connected inverters. The presented method can deal effectively with unexpected parameter variations and vague meddling, thereby authenticating the maximum power output of the PV system [46]. For the control of PV converter in a grid, it is recommended to introduce mu-synthesis into $\mathrm{H}$-infinity approach. The proposed scheme rejects system ambiguities, such grid impedance varieties and voltage harmonics; the outcome system exhibits undistorted response, particularly in the face of harsh nonlinearities [47]. As a result, these advanced nonlinear methods have revealed creditable performance, and also furnishes the prospection of further investigation.

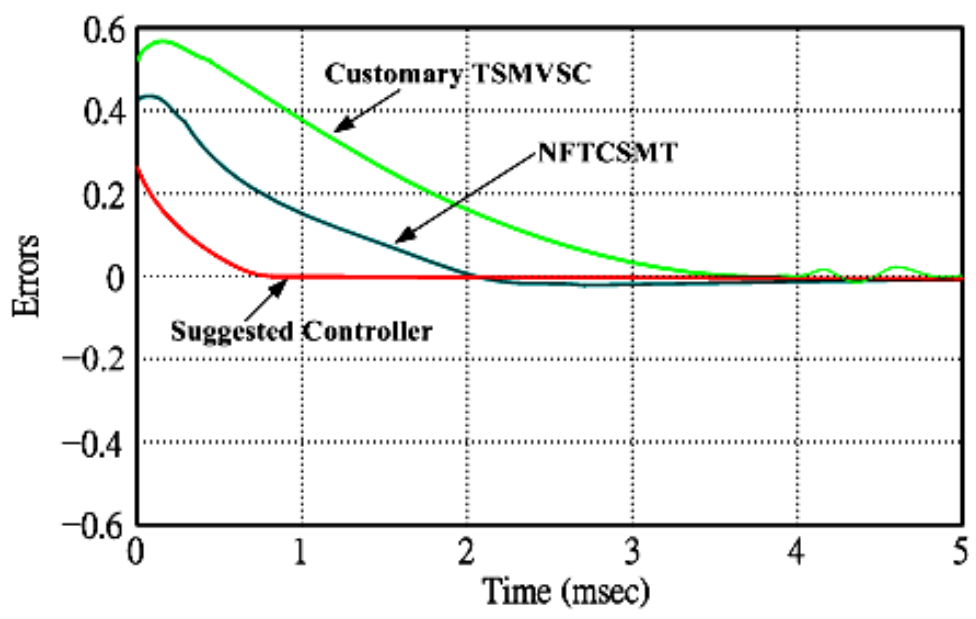

Figure 11. The confrontation of optimization results using diverse methods.

\section{Conclusions}

In the presence of severe nonlinear rectified loading as well as step-loading variations, the NFTCSMT based on improved QPSO is developed for REIs in this paper. The significance of TSMVSC lies in: (1) rapider state fixed/limited convergent response and (2) existing in non-singularity. In addition, the optimal parameters of the NFTCSMT are selected by improved QPSO, so as to get following benefits: (1) the abatement of the dither, (2) the acceleration of the convergence speed, and (3) globally convergent stability. The determination of these optimal parameters solves the difficulty in determining customary parameters, which uses repeated experiments with very tediousness as well as inefficiency. The Lyapunov-function candidate analyzes fixed/limited-time stabilization with respect to the suggested controller. The fixed/limited-time sliding-manifold reachability, fixed/limited-time feedback system stability and fixed/limited-time trajectory tracking are corroborated. Simulations and experimentation results exhibit that the suggested REI possesses good performance in smaller than voltage THD and dip, which exceed specified IEEE standards under diverse load situations. As a result, the suggested controller will inspire the author to realize closed-loop architectures of other new circuits, for instance a high step-up converter of wind energy system, detection circuit of 
ground insulation fault, and LED street light driver [48-50]. The author is also convinced that the renewable energy correlative circuits (both an AC-DC rectifier and DC-DC converter) can definitely be integrated into the presented REI, generating more contributions in control of power electronic converters. The above-mentioned circuits will facilitate more research and exploration of the author in prospective work.

Funding: This research was funded by the Ministry of Science and Technology (MOST) of Taiwan, R.O.C., under contract MOST 107-2221-E-214-006.

Acknowledgments: The author gratefully acknowledges the financial support of the Ministry of Science and Technology of Taiwan, R.O.C., under project number MOST 107-2221-E-214-006.

Conflicts of Interest: The author declares that there is no conflict of interest regarding the publication of this article.

\section{References}

1. Chen, X.; Zhong, Y.; Zhang, L.; Howarter, J.A.; Baba, A.A.; Wang, C.; Sun, Z.; Zhang, M.; Olivetti, E.; Luo, A.; et al. Energy Technology 2020: Recycling, Carbon Dioxide Management, and Other Technologies; Springer International Publishing: New York, NY, USA, 2020.

2. Belu, R. Renewable Energy Systems: Fundamentals and Source Characteristics; CRC Press: Boca Raton, FL, USA, 2019.

3. Bizon, N. Optimization of the Fuel Cell Renewable Hybrid Power Systems; Springer International Publishing: New York, NY, USA, 2020.

4. Jenkins, N.; Ekanayake, J. Renewable Energy Engineering; Cambridge University Press: Cambridge, UK, 2017.

5. Bose, B.K. Power Electronics in Renewable Energy Systems and Smart Grid: Technology and Applications; John Wiley \& Sons: Hoboken, NJ, USA, 2019.

6. Suzuki, T.; Inagaki, S.; Susuki, Y.; Tran, A.T. Design and Analysis of Distributed Energy Management Systems; Springer International Publishing: New York, NY, USA, 2020.

7. Liu, J.; Gao, Y.; Yin, Y.; Wang, J.; Luo, W.; Sun, G. Sliding Mode Control Methodology in the Applications of Industrial Power Systems; Springer International Publishing: New York, NY, USA, 2020.

8. Ryvkin, S.E.; Lever, E.P. Sliding Mode Control for Synchronous Electric Drives; John Wiley \& Sons: Hoboken, NJ, USA, 2019.

9. Steinberger, M.; Horn, M.; Fridman, L. Variable-Structure Systems and Sliding-Mode Control; Springer International Publishing: New York, NY, USA, 2020.

10. Argha, A.; Su, S.; Li, L.; Nguyen, H.T.; Celler, B.G. Advances in Discrete-Time Sliding Mode Control: Theory and Applications; John Wiley \& Sons: Hoboken, NJ, USA, 2018.

11. Feshara, H.F.; Ibrahim, A.M.; El-Amary, N.H.; Sharaf, S.M. Performance Evaluation of Variable Structure Controller Based on Sliding Mode Technique for a Grid-Connected Solar Network. IEEE Access 2019, 7, 84349-84359. [CrossRef]

12. Alali, M.A.E.; Shtessel, Y.B.; Barbot, J.P. Grid-Connected Shunt Active LCL Control via Continuous Sliding Modes. IEEE/ASME Trans. Mechatron. 2019, 24, 729-740. [CrossRef]

13. Shahab, M.A.; Mozafari, B.; Soleymani, S.; Dehkordi, N.M.; Shourkaei, H.M.; Guerrero, J.M. Distributed Consensus-Based Fault Tolerant Control of Islanded Microgrids. IEEE Trans. Smart Grid. 2020, 11, 37-47. [CrossRef]

14. Ortega, R.; Carranza, O.; Rodríguez, J.J.; García, V.H.; Sosa, J.C.; Alvarado, J.M. Development and Application of a Reconfigurable Photovoltaic Inverter for Operation within a Microgrid. IEEE Access 2019, 7, 98755-98770. [CrossRef]

15. Guo, B.; Su, M.; Sun, Y.; Wang, H.; Dan, H.B.; Tang, Z.T.; Cheng, B. A Robust Second-Order Sliding Mode Control for Single-Phase Photovoltaic Grid-Connected Voltage Source Inverter. IEEE Access 2019, 7 , 53202-53212. [CrossRef]

16. Cxakar, O.; Tanyýldýzý, A.K. Application of moving sliding mode control for a DC motor driven four-bar mechanism. Adv. Mech. Eng. 2018, 10, 1-13.

17. Shao, S.Y.; Chen, M. Sliding-mode-disturbance-observer-based adaptive neural control of uncertain discrete-time systems. Sci. China Inf. Sci. 2019, 63, 1-3. [CrossRef] 
18. Yan, H.C.; Zhou, X.P.; Zhang, H.; Yang, F.W.; Wu, Z.G. A Novel Sliding Mode Estimation for Microgrid Control with Communication Time Delays. IEEE Trans. Smart Grid. 2019, 10, 1509-1520. [CrossRef]

19. Wang, Z.; Li, S.H.; Li, Q. Discrete-Time Fast Terminal Sliding Mode Control Design for DC-DC Buck Converters with Mismatched Disturbances. IEEE Trans. Ind. Inf. 2020, 16, 1204-1213. [CrossRef]

20. Jia, S.Y.; Shan, J.J. Finite-Time Trajectory Tracking Control of Space Manipulator Under Actuator Saturation. IEEE Trans. Ind. Electron. 2020, 67, 2086-2096. [CrossRef]

21. Qiao, J.Z.; Li, Z.X.; Xu, J.W.; Yu, X. Composite Nonsingular Terminal Sliding Mode Attitude Controller for Spacecraft with Actuator Dynamics Under Matched and Mismatched Disturbances. IEEE Trans. Ind. Inf. 2020, 16, 1153-1162. [CrossRef]

22. Rascón, R.; Rosas, D.I.; Rodríguez-Quiñonez, J.C. Robust Continuous Control for a Class of Mechanical Systems Based on Nonsingular Terminal Sliding Mode. IEEE Access 2020, 1-9. [CrossRef]

23. Mishra, J.P.; Li, C.J.; Jalili, M.; Yu, X.H. Robust Second-Order Consensus Using a Fixed-Time Convergent Sliding Surface in Multiagent Systems. IEEE Trans. Cybern. 2020, 50, 846-855. [CrossRef]

24. Fang, Y.M.; Zhu, Y.K.; Fei, J.T. Adaptive Intelligent Sliding Mode Control of a Photovoltaic Grid-Connected Inverter. Appl. Sci. 2018, 8, 1756. [CrossRef]

25. Mobayen, S. Adaptive global sliding mode control of underactuated systems using a super-twisting scheme: An experimental study. J. Vib. Control. 2019, 25, 2215-2224. [CrossRef]

26. Yang, T.; Gao, X.H. Adaptive Neural Sliding-Mode Controller for Alternative Control Strategies in Lower Limb Rehabilitation. IEEE Trans. Neural Syst. Rehabil. Eng. 2020, 28, 238-247. [CrossRef] [PubMed]

27. An, Q.T.; Zhang, J.Q.; An, Q.; Liu, X.G.; Shamekov, A.; Bi, K.T. Frequency-Adaptive Complex-Coefficient Filter-Based Enhanced Sliding Mode Observer for Sensorless Control of Permanent Magnet Synchronous Motor Drives. IEEE Trans. Ind. Appl. 2020, 56, 335-343. [CrossRef]

28. Liu, Y.J.; Chen, H. Adaptive Sliding Mode Control for Uncertain Active Suspension Systems with Prescribed Performance. IEEE Trans. Syst. ManCybern. Syst. 2020, 1-9. [CrossRef]

29. Mao, Q.; Dou, L.Q.; Yang, Z.S.; Tian, B.L.; Zong, Q. Fuzzy Disturbance Observer-Based Adaptive Sliding Mode Control for Reusable Launch Vehicles with Aeroservoelastic Characteristic. IEEE Trans. Ind. Inform. 2020, 16, 1214-1223. [CrossRef]

30. Ye, M.; Wang, H. A Robust Adaptive Chattering-Free Sliding Mode Control Strategy for Automotive Electronic Throttle System via Genetic Algorithm. IEEE Access 2020, 8, 68-80. [CrossRef]

31. Jiang., B.P.; Karimi, H.R.; Kao, Y.G.; Gao, C.C. Adaptive Control of Nonlinear Semi-Markovian Jump T-S Fuzzy Systems with Immeasurable Premise Variables via Sliding Mode Observer. IEEE Trans. Cybern. 2020, 50, 810-820. [CrossRef] [PubMed]

32. Cheng, L.; Zhu, Z.C.; Shen, G.; Wang, S.J.; Li, X.; Tang, Y. Real-Time Force Tracking Control of an Electro-Hydraulic System Using a Novel Robust Adaptive Sliding Mode Controller. IEEE Access 2020, 8 , 13315-13328.

33. Zhang, Z.N.; Niu, Y.G.; Song, J. Input-to-State Stabilization of Interval Type-2 Fuzzy Systems Subject to Cyberattacks: An Observer-Based Adaptive Sliding Mode Approach. IEEE Trans. Fuzzy Syst. 2020, 28, 190-203. [CrossRef]

34. Zhang, Y.; Zeng, T.Y.; Li, G. Robust Excitation Force Estimation and Prediction for Wave Energy Converter M4 Based on Adaptive Sliding-Mode Observer. IEEE Trans. Ind. Inform. 2020, 16, 1163-1171. [CrossRef]

35. Chen, Z.; Huang, F.G.; Chen, W.J.; Zhang, J.H.; Sun, W.C.; Chen, J.W.; Gu, J.; Zhu, S.Q. RBFNN-Based Adaptive Sliding Mode Control Design for Delayed Nonlinear Multilateral Telerobotic System with Cooperative Manipulation. IEEE Trans. Ind. Inform. 2020, 16, 1236-1247. [CrossRef]

36. Haq, I.; Khan, Q.; Khan, I.; Akmeliawati, R.; Nisar, K.S.; Khan, I. Maximum power extraction strategy for variable speed wind turbine system via neuro-adaptive generalized global sliding mode controller. IEEE Access 2020, 1-13. [CrossRef]

37. Li, R.G.; Wu, H.N. Adaptive synchronization control with optimization policy for fractional-order chaotic systems between 0 and 1 and its application in secret communication. Isa Trans. 2019, 92, 35-48. [CrossRef]

38. Rehman, O.U.; Rehman, S.U.; Tu, S.S.; Khan, S.; Waqas, M.; Yang, S.Y. A Quantum Particle Swarm Optimization Method with Fitness Selection Methodology for Electromagnetic Inverse Problems. IEEE Access 2018, 6, 63155-63163. [CrossRef] 
39. Rehman, O.U.; Yang, S.Y.; Khan, S.; Rehman, S.U. A Quantum Particle Swarm Optimizer with Enhanced Strategy for Global Optimization of Electromagnetic Devices. IEEE Trans. Ind. Inform. 2019, 55, 1-4. [CrossRef]

40. $\mathrm{Mu}, \mathrm{L}$. Dynamic scale quantum-inspired optimisation algorithm under harmonic oscillator potential well. Electron. Lett. 2019, 55, 1012-1015. [CrossRef]

41. Chakraborty, R.; Sushil, R.; Garg, M.L. ICQPSO-based multilevel thresholding scheme applied on colour image segmentation. IET Signal Process. 2019, 13, 387-395. [CrossRef]

42. Sharma, C.P.; Sapkota, K.; Tamang, D.B.; Newpaney, S.; Bhutia, J.N. Harmonic analysis and comparison between single phase three-level pulse width modulation (PWM) inverter and sinusoidal pulse width modulation (SPWM) inverter implementing analog circuits. In Proceedings of the 2019 Second International Conference on Advanced Computational and Communication Paradigms (ICACCP), Gangtok, India, 25-28 February 2019.

43. Zhang, B.; Qiu, D. m-Mode SVPWM Technique for Power Converters; Springer: Singapore, 2019.

44. Zhang, X.N.; Foo, G.H.B.; Jiao, T.; Ngo, T.; Lee, C.H.T. A Simplified Deadbeat Based Predictive Torque Control for Three-Level Simplified Neutral Point Clamped Inverter Fed IPMSM Drives Using SVM. IEEE Trans. Energy Convers. 2019, 34, 1906-1916. [CrossRef]

45. Fuentes, G.A.R.; Ruget, R.I.; Castello, R.C. Robust repetitive control of power inverters for standalone operation in DG systems. IEEE Trans. Energy Convers. 2019. [CrossRef]

46. Yang, T.G.; Cai, Z.H.; Xun, Q. Adaptive Backstepping-Based $\mathrm{H}_{\infty}$ Robust Controller for Photovoltaic GridConnected Inverter. IEEE Access 2020, 8, 17263-17272. [CrossRef]

47. Ashtiani, N.A.; Azizi, S.M.; Khajehoddin, S.A. Robust Control Design for High-Power Density PV Converters in Weak Grids. IEEE Trans. Control Syst. Technol. 2019, 27, 2361-2373. [CrossRef]

48. Yang, L.S.; Lin, C.C.; Chang, E.C. A Three-Phase AC-DC High Step-up Converter for Microscale Wind-power Generation Systems. J. Power Electron. 2016, 16, 1861-1868. [CrossRef]

49. Liu, Y.C.; Chang, E.C.; Lee, Y.L.; Lin, Y.Z. Detection of Ground Insulation Faults in Ungrounded DC Power Supply Systems by Using a DC Current Injection Method. Energy Procedia. 2019, 156, 349-355. [CrossRef]

50. Cheng, C.A.; Chang, C.H.; Cheng, H.L.; Chang, E.C.; Chung, T.Y.; Chang, M.T. A Single-Stage LED Streetlight Driver with Soft-Switching and Interleaved PFC Features. Electronics 2019, 8, 911. [CrossRef] 